\title{
Comunicação terapêutica no cuidado pré-operatório de mastectomia
}

\author{
Therapeutic communication in perioperative care of mastectomy
}

Comunicación terapéutica en la atención pre-operatoria de mastectomía

\section{Miria Conceição Lavinas Santos', Francisco Stélio de Sousa', Pricilla Cândido Alves, Isabela Melo Bonfim', Ana Fátima Carvalho Fernandes'}

'Universidade Federal do Ceará. Departamento de Enfermagem. Programa de Pós-graduação. Fortaleza, CE

Submissão: 28/04/2009

Aprovação: 18/05/2010

\section{RESUMO}

Relato de experiência sobre a prática docente-assistencial em hospital de oncologia, com o propósito refletir sobre o desenvolvimento da comunicação terapêutica do (a) enfermeiro (a) na unidade de internação pré-operatória de mulheres mastectomizadas. Constatouse Que o exercício da comunicação terapêutica configura-se uma prática favorável, porQuanto estabelece o vínculo enfermeiro-paciente, sendo a paciente produtora do próprio cuidado. Compreendendo a fragilidade da paciente nesta fase, o (a) enfermeiro (a) assume compromisso de preestabelecer os cuidados de enfermagem em parceria, de forma a atender às necessidades da paciente, e com vistas, principalmente, à autonomia do cuidado. Diante disto, apresenta-se uma proposta de assistência de enfermagem gerenciada e fundamentada em uma relação recíproca de cuidado participativo e humanizante.

Descritores: Comunicação; Enfermagem; Cuidados pré-operatórios; Mastectomia.

\section{ABSTRACT}

The authors report their experience as teacher-nurse for years in oncologic hospital with the aim of enabling reflection on the development of nursing therapeutic communication at the hospital preoperative unit for mastectomized women. It was found that the practice of therapeutic communication becomes a favorable practice, because establish the nurse-patient relationship, with patient producing his own care. Understanding the fragility of the patient at this stage, the nurse takes commitment to predefine the nursing care in partnership, in order to meet the patient needs, aiming to, mainly, the autonomy of care. In this way, the authors present a proposal for nursing managed care based on a mutal relationship, human and participatory care.

Key words: Communication; Nursing; Preoperative care; Mastectomy.

\section{RESUMEN}

Relato de experiencia sobre la práctica docente-asistencial en el hospital de oncología con el fin de reflexionar sobre el desarrollo de la terapia del enfermero en el hospital preoperatorio de las mujeres mastectomizadas. Se ha señalado Que la práctica de la comunicación terapéutica establece una práctica favorable, establece una relación enfermero-paciente, Quedándose la paciente como la productora de la propia atención. La comprensión de la fragilidad de la paciente en esta etapa, el enfermero tiene el compromiso previamente a los cuidados de enfermería en colaboración con el fin de satisfacer a las necesidades de la paciente, y su objetivo principal, la autonomía de la atención. Frente a esto, se presenta una propuesta de atención de enfermería y gerenciada basada en una relación de mutuo cuidado participativo y humanizante.

Descriptores: Comunicación; Enfermería; Cuidados preoperatorios; Mastectomía. 


\section{INTRODUÇÃO}

Atualmente, aos avanços científicos e técnicos na área da mastologia permitem melhorar os índices de cura de mulheres acometidas pelo câncer de mama, contribuindo para o aumento gradativo da expectativa de vida dessas mulheres.

No entanto, concomitantemente a este desenvolvimento, nos deparamos com número significativo de mulheres acometidas com esta neoplasia, em fase avançada, carecendo de hospitalização e cirurgia, na eual, para a grande maioria, é mutiladora.

A necessidade da hospitalização e o diagnóstico não esperado de câncer de mama ocasionam desestabilidades físicas, emocionais, sociais e espirituais ${ }^{(1-4)}$.

Uma vez enferma, a mulher com câncer de mama começa a vivenciar uma situação indesejável, e para a Qual, geralmente, jamais estará preparada: vê ocorrer gradativamente a perda de suas possibilidades como pessoa produtiva, particularmente, em relação ao seu papel de mãe, esposa e mulher. Neste contexto, percebe-se dentre os efeitos psicológicos a não aceitação do diagnóstico.

A mulher portadora de câncer de mama traspassa por seis lutos: a existência da possibilidade de ter câncer; o diagnóstico; o tratamento cirúrgico; a perda da imagem corporal; as limitações em decorrência da cirurgia e os tratamentos neoadjuvantes ${ }^{(5-7)}$.

Ao longo da prática acadêmico-profissional, percebeu-se Que no primeiro momento, o luto via tratamento cirúrgico é reconfortante, ante a sensação de alívio de ter sobrevivido à cirurgia e a esperança de estar curada. Em seguida, ele é acompanhado de enfrentamento, de desconfortos pré-operatórios, principalmente no referente à própria incisão e à presença de drenos, denunciando a necessidade de cuidados de enfermagem.

Estudos realizados reconhecem que o cuidado de enfermagem a mulher no período pré-operatório não abrange somente o atendimento das necessidades fisiológicas, mas envolve os valores pessoais e o modo como ela se sente como mulher portadora de um câncer de mama, os Quais refletem, sobretudo em seu pósoperatório $^{(6-8)}$.

Como evidenciado, a literatura é abundante em estudos sobre os aspectos psicológicos presentes no câncer de mama ${ }^{(9-11)}$, mas insuficiente sobre os cuidados de enfermagem envolvendo a fase cirúrgica neste tipo de câncer ${ }^{(6,8,12)}$.

A par dessa realidade, compreende-se a possibilidade de relatar a prática no cuidado pré-operatório à mulher com câncer de mama, cujo cuidado é estabelecido com um envolvimento recíproco, no sentido de restabelecer a saúde sob a ótica da mulher.

Diante dessas considerações, é conduzida a reflexão contida neste artigo Que objetiva refletir acerca do cuidado pré-operatório da mulher portadora de câncer de mama, baseada na comunicação terapêutica como referência do cuidar pelo(a) enfermeiro(a) atuante na área oncológica.

\section{COMUNICAÇÃO TERAPÊUTICA NO CUIDADO PRÉ- OPERATÓRIO DE MASTECTOMIA}

O estudo foi realizado em um hospital filantrópico de referência em oncologia, do estado do Ceará, Brasil. Esta instituição constitui um hospital de assistência terciária, fundado em 1944, como entidade filantrópica. Tem como missão básica: prevenir, diagnosticar e tratar o câncer, de forma efetiva, eficiente e humanizada. Conta atualmente com 140 leitos distribuídos entre as clínicas: médica e cirúrgica. Em sua maioria, os atendimentos são prestados pelo Sistema Único de Saúde (SUS), mas o hospital também atende a convênios e particulares, com predomínio na atenção às doenças crônico-degenerativas, em especial o câncer, e vem se destacando como um dos centros de referência para o diagnóstico e tratamento de pacientes oncológicos.

Os clientes têm acesso aos serviços assistenciais, tais como: centro de diagnóstico por imagem; Quimioterapia; radioterapia; centro-cirúrgico; sala de recuperação anestésica; unidade de terapia intensiva; banco de sangue; laboratório de anatomia patológica; imunohistoquímica e análises clínicas; unidades de internação (apartamentos e enfermarias) e oncologia pediátrica com todos os demais serviços direcionados à criança. Além disso, o hospital disponibiliza de consultórios médicos, atendimento psicológico, assistência social, setor de registro de câncer, biblioteca, setor de expurgo, central de material, velório, farmácia e lanchonete, dentre outros setores de grande importância.

$\mathrm{O}$ (a) enfermeiro(a) é presença indispensável na equipe multiprofissional, por estabelecer com a mulher acometida pelo câncer de mama uma comunicação terapêutica Que proporciona cuidado de enfermagem que, por sua vez, atende às expectativas e necessidades da mulher, assegurando conforto físico, emocional e espiritual.

A comunicação terapêutica é a utilização da competência interpessoal, com vistas a atender às necessidades do paciente em todas as suas dimensões, considerando a sua cultura, o ambiente e o seu ser. É a habilidade do profissional em usar o seu conhecimento sobre comunicação, estabelecendo uma relação efetiva e consciente com o paciente, de modo a ajudá-lo a enfrentar a tensão temporária, a conviver com outras pessoas, a ajustar-se ao Que não pode ser mudado, a superar os bloqueios à autorealização ${ }^{(13)}$.

Por meio das trocas materiais e afetivas, cria-se uma rede de negociação, a Qual aumenta a participação da mulher e a compreensão desta sobre a conQuista de sua reabilitação psicossocial.

Contudo, a vulnerabilidade do estresse emocional, no préoperatório, é sintoma de alta incidência na mulher portadora de câncer de mama. Os Quadros ansiosos aparecem em conseQuência do próprio diagnóstico ou imediatamente após a cirurgia mutiladora $^{(5)}$.

Diante desses casos, a fonte de apoio estabelecida pela comunicação terapêutica torna-se peça imprescindível, bem como o apoio em Deus, na família e na equipe multiprofissional, conforme experiência com mulheres com diagnóstico de câncer de mama.

Logo, o(a) enfermeiro(a) responsável pelo cuidado pré-operatório da mulher com câncer de mama na unidade de internação e, principalmente, dąuela Que precisa se submeter à cirurgia, devendo ser planejado e estabelecido metas em face dos seguintes desconfortos: reações emocionais relacionadas ao diagnóstico de câncer; déficit de conhecimento sobre o câncer de mama e opções de tratamento; medo relacionado aos tratamentos específicos; alterações da imagem corporal ou possível morte; aceitação ineficaz (individual e/ familiar) do diagnóstico; dor e desconforto pósoperatório.

Planejar o cuidado a partir da existência desse conjunto de 
preocupações e desconfortos é o primeiro passo para se estabelecer a interação, possibilitando o desenvolvimento e a sustentação do Que se julga ser comunicação terapêutica.

Pois, somente existe a comunicação terapêutica porQue existe a interação, Que envolve troca, colaboração e compartilhamento de informações expelidas e recebidas entre os sujeitos envolvidos no cuidado (paciente $x$ enfermeiro). Nesta relação de ajuda, o (a) enfermeiro (a) e a paciente crescem e se fortalecem em aprendizado mútuo.

A comunicação terapêutica estimula a paciente a aprender, entender e a buscar a resolução para os seus desconfortos, mediante a construção e reestruturação de informações estimuladas pela interação. Por meio dela, o(a) enfermeiro(a) compreende a paciente e a maneira como ela enxerga, sente e percebe e age no mundo. A partir desta compreensão, o(a) enfermeiro(a) poderá identificar os problemas da paciente e o significado Que esta lhes atribui, e dessa forma estabelecer uma comunicação para uma ação terapêutica ${ }^{(14)}$.

Por conseguinte, a meta do(a) enfermeiro(a) na fase préoperatória deverá ser amenizar os desconfortos, implementando medidas Que reduzam o medo e ansiedade pré e pós-operatória para abrandar a capacidade de enfrentamento, promovendo a capacidade de tomar decisões, a fim de estabelecer um tratamento fisiológico com vistas ao alívio da dor e à prevenção de complicações, além de melhorar o autoconceito.

Portanto, acredita-se Que a comunicação terapêutica propiciará o estabelecimento de intervenções significantes/significativas na compreensão e no enfrentamento, pela mulher, das diferentes fases do processo de tratamento e de novas ordenações para a sua vida.

As mulheres, ao reconhecerem a preocupação consigo mesmas, buscam soluções para melhorar o seu estado de saúde. Corroboramos essas opiniões, pois o convívio com estas mulheres possibilitou acompanhar a evolução desse processo ${ }^{(12)}$.

Dessa forma, o processo do adoecer é um momento de autoorganização, sobretudo, por envolver uma experiência de desordem. Tal experiência adQuire um sentido específico no momento existencial dessa mulher, com significados Que os sintomas, as experiências com o tratamento e as relações passam a ter no contexto de sua vida.

Partindo-se dessa premissa, se existir a comunicação terapêutica, instrumento essencial do cuidado de enfermagem ${ }^{(15)}$, há o envolvimento do comportamento recíproco. Com isto, observa-se também Que o pós-operatório será menos estressante em virtude do estabelecimento da interação enfermeira-mulher, interação esta Que induz a mulher a mencionar os desconfortos de menor a maior intensidade. Assim, se estabelecerá o já mencionado efeito da comunicação terapêutica.
É importante também ressaltar Que a Quantidade e a regulação temporal das informações transmitidas têm contribuído para um pós-operatório menos estressante, em Que a mulher otimiza a capacidade de enfrentamento e inicia o autocuidado. A satisfação em relação ao resultado é evidente no cuidado com a ferida operatória (integridade cutânea); no manuseio do dreno de portvac, alvo de muita apreensão; nos exercícios com o braço afetado, orientação fisioterápica; e no alívio e o desconforto da dor.

Nessa perspectiva, o diálogo permite Que o cliente expresse suas ideias e opiniões, ao mesmo tempo em que estabelece com a equipe de enfermagem uma relação Que implica em uma resposta humana positiva, ou seja, em um cuidado participativo e humanizante $\mathrm{e}^{(16-18)}$

A proximidade com esta mulher mediante cuidado de enfermagem envolvendo a comunicação terapêutica, favore a capacidade de enfrentamento, a promoção e a participação nos cuidados de forma compartilhada. Portanto, permite o preparo para a alta e o estabelecimento, principalmente do cuidado continuado com a perspectiva de adesão da mulher ao acompanhamento multiprofissional.

\section{CONSIDERAÇÕES FINAIS}

A proposta de comunicação terapêutica aQui discutida permite romper com o paradigma tradicional da intervenção de enfermagem e internação hospitalar, Que se mostram promotores de exclusão da paciente na participação da organização do autocuidado.

A equipe de enfermagem exerce papel fundamental no processo de recuperação da mulher mastectomizada, uma vez Que esta promove suporte emocional e informativo sobre os cuidados necessários à reabilitação pós-mastectomia, além de proporcionar tranquilidade e conforto perante os sentimentos e as expectativas. Cabe, ainda, ao(à) enfermeiro(a) orientar para a alta e direcionar a mulher para o autocuidado e para grupos Que promovam a reintegração à sociedade e a seu cotidiano familiar.

Com reforço neste estudo, considera-se necessário agregar a experiência das autoras a de outros(as) enfermeiros(as) Que prestam cuidado de enfermagem à mulher com câncer nas unidades de internação.

Essa estratégia se apresenta como um modelo de transformação no planejamento de cuidado, significando a garantia de conquistas para a mulher Que vivencia o pré e pós-operatório de câncer de mama. Coneuistas voltadas a uma concepção positiva do restabelecimento da saúde com Qualidade, revelando-se parceria bastante positiva entre enfermeiros (as) e mulher com câncer de mama.

\section{REFERÊNCIAS}

I. Coelho MS, Sampaio MSB, Pereira ER, Martins CC, Silva RMCRA, Medeiros CL. Mulheres mastectomizadas: uma proposta de cuidado de si com base nas concepções de Michel Foucault. Rev Enferm UFPE On Line 2010; 4(1):311-18.

2. Bittencourt JFV, Cadete MMM. Vivências da mulher a ser mastectomizada: esclarecimentos e orientação. Rev Bras Enferm 2002; 55(4): 420-23.

3. Andolhi R, Guido LA, Bianchi ERF. Stress e coping no período perioperatório de câncer de mama. Rev Esc Enferm USP 2009;

43(3):71 I-20.

4. Pinho LS, Campos ACS, Fernandes AFC, Lôbo SA. Câncer de mama: da descoberta à recorrência da doença. Rev Eletron Enferm 2007; 9(1): 154-65.

5. Maluf MFM, Mori LJ, Barros ACSD. O impacto psicológico do câncer de mama. Rev Bras Cancerol 2005; 5 I (2): 149-54.

6. Fernandes AFC, Santos MCLS, Silva RM. Câncer de mama: como detectar e cuidar. Fortaleza (CE): UFC; 2005.

7. Fernandes AFC, Araújo IMA. Enfrentando o diagnóstico de 
câncer de mama- depoimentos de mulheres mastectomizadas. Fortaleza (CE): UFC; 2005.

8. Oliveira MS, Fernandes AFC, Melo EM, Barbosa ICFI. Cuidados preoperatorios de mastectomia bajo la óptica de la mujer. Rev Cubana Enfermer 2005; 2 I (2).

9. Barreto RAS, Suzuki K, Lima MA, Moreira AA. As necessidades de informação de mulheres mastectomizadas subsidiando a assistência de enfermagem. Rev Eletron Enferm 2008; I0(I): I10-23.

10. Backaus VP. Psychiatric aspects of breast cancer. Harv Rev Psychiatr 2002; 10(5): 307-14.

1 I. Cohen L, Hack TF, Moor C, Katz J, Goss PE. The effects of type of sugery and time o psychological adjustment in woman after breast cancer treatment. Ann Surg Oncol 2000; 7(6): 427-34.

12. Ferreira MLSM, Mamede MV. Representação do corpo na relação consigo mesma após mastectomia. Rev Latino-am Enfermagem 2003; II (3): 299-304.
13. Stefanelli MC. Comunicação com o paciente-teoria e ensino. São Paulo: Robe; 1993.

14. Stefanelli MC. Introdução à comunicação terapêutica. In: Stefanelli MC, Carvalho MC, organizadores. A comunicação nos diferentes contextos da enfermagem. Barueri: Manole: 2005. p.62-72.

15. Pontes AC, Leitão IMTA, Ramos IC. Comunicação terapêutica em enfermagem: instrumento essencial do cuidado. Rev Bras Enferm 2008; 6I(3):312-18.

16. Barcelos LMS, Alvim NAT. Conversa: um cuidado fundamental de enfermagem na perspectiva do cliente hospitalizado. Rev Bras Enferm 2003; 56(3):236-4l.

17. Naranjo BIC, Ricaute GGP. La comunicacíon com los pacientes. Invest Educ Enferm 2006; 24(I):94-8.

18. López LM, Carvalho EC. La comunicación terapéutica durante instalación de venoclisis: uso de la simulación filmada. Rev Latino-am Enfermagem 2006; 14(5):658-65. 